At day 15, following a 14-day titration period, 92.3\% (60/65) were receiving the recommended dose of $2403 \mathrm{mg} /$ day pirfenidone. One patient had discontinued treatment. Data was available for 53 patients at 3 months; 42 (79.2\%) continued to receive pirfenidone, whilst 11 (20.8\%) had stopped therapy. Between 3 and 6 months, $93.1 \%(27 / 29)$ continued pirfenidone with only 2 further patients (6.9\%) stopping treatment.

Of the 68 patients, 30 (44.1\%) experienced an adverse drug reaction (ADR) resulting in a change of dose or cessation of pirfenidone. The commonest ADRs were GI-upset and photosensitive rash. Of those experiencing ADRs, with data available, 12/22 (54.5\%) continued pirfenidone after a dose-reduction. In 53 patients for whom pre-treatment liver function tests were available, only two showed a minor increase above the upper limit of normal. During the observation period $10.3 \%(7 / 68)$ of patients suffered an acute exacerbation. Collection of data to determine effect of pirfenidone on lung function at 6 months of treatment is on-going.

Conclusions Patients enrolled in the NPP are demographically representative of the UK IPF population but have moderately severe disease that is on average more advanced than that seen in the CAPACITY study. Pirfenidone treatment is reasonably well tolerated. On-going observation of patients enrolled in the UK NPP should provide invaluable insights into the real-world benefits of pirfenidone in individuals with IPF.

\section{S99 A PROSPECTIVE EVALUATION OF SERIAL LUNG FUNCTION IN A UK COHORT OF PATIENTS WITH LYMPHANGIOLEIOMYOMATOSIS (LAM)}

doi:10.1136/thoraxjnl-2012-202678.104

IV Navaratnam, ${ }^{2} \mathrm{O}$ Gbolahan, 'RB Hubbard, ${ }^{2} \mathrm{SR}$ Johnson. 'Division of Respiratory Medicine, University of Nottingham, Nottingham, UK; '2Division of Therapeutics and Molecular Medicine, University of Nottingham, National Centre for Lymphangioleiomyomatosis, Nottingham, UK

Background LAM is a rare disease of women in which abnormal smooth muscle cells invade and destroy particularly the lungs and lymphatics. We wanted to relate the progression of the disease with presenting clinical features and to determine rate of decline of lung function in patients to improve prognostic predictions and stratify patients for clinical trials.

Methods Clinical features and pulmonary function test (PFT) data were collected from 125 patients with a definitive diagnosis of LAM by European Respiratory Society Criteria receiving care at the National Centre for LAM. Ethical approval for use of patient data was obtained and patients gave informed consent. 74 of these patients with more than two sets of PFTs formed the prospective cohort. Patients were grouped into degree of lung function impairment based on $\mathrm{FEV}_{1}$ or $\mathrm{DL}_{\mathrm{CO}}$ at first presentation: unimpaired; PFTs $>80 \%$ predicted, mild; $61 \%-80 \%$, moderate; $40 \%-60 \%$, severe; $<40 \%$.

Results The median follow-up period was 28.5 months (range 3-262). PFTs declined as the disease progressed (figure shows all prospective $\mathrm{FEV}_{1}$ measurements from presentation). Mean changes for $\mathrm{FEV}_{1}$ were $-49.68 \mathrm{ml} / \mathrm{yr}, \mathrm{FVC}-44 \mathrm{ml} / \mathrm{yr}$, and $\mathrm{DL}_{\mathrm{CO}}-0.07 \mathrm{mmol} /$ $\mathrm{min} / \mathrm{kPa} / \mathrm{yr}$. Rate of change in $\mathrm{FEV}_{1}$ varied between patients with different degrees of lung function impairment at first presentation with change in $\mathrm{FEV}_{1}$ within the groups being: unimpaired $-84 \mathrm{ml} /$ $\mathrm{yr}$, mild $-32 \mathrm{ml} / \mathrm{yr}$, moderate $-59 \mathrm{ml} / \mathrm{yr}$ and severe $-24 \mathrm{ml} / \mathrm{yr}$.

Conclusion The rate of decline of FEV1 in this cohort was slower than in previous retrospective studies of patients with LAM. Change in $\mathrm{FEV}_{1}$ varied with degree of lung function impairment being more rapid in those with early disease ie. well preserved lung function at diagnosis. Clinical features at onset may be associated with rate of progression of LAM. These data may be helpful in advising patients on prognosis and designing clinical trials.

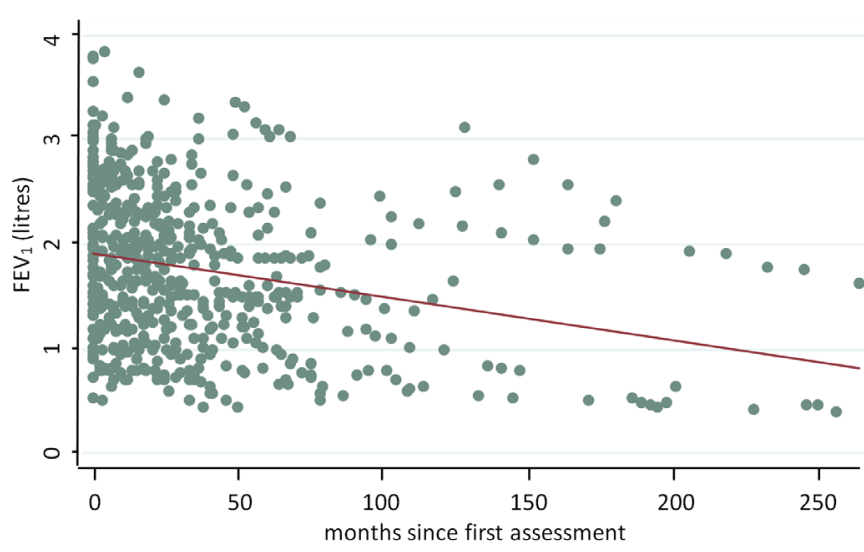

Abstract S99 Figure 1

\section{S100 NATURAL HISTORY OF RENAL ANGIOMYOLIPOMA IN SPORADIC AND TUBEROUS SCLEROSIS COMPLEX ASSOCIATED LYMPHANGIOLEIOMYOMATOSIS: IMPLICATIONS FOR CLINICAL CARE}

doi:10.1136/thoraxjnl-2012-202678.105

ZW Yeoh, V Navaratnam, RB Hubbard, SR Johnson. Therapeutics and Molecular Medicine, Respiratory Medicine and National Centre for LAM, Unversity of Nottingham, Nottingham, UK

Background Lymphangioleiomyomatosis (LAM) is a rare disease of women categorised by lung cysts, lymphatic abnormalities and angiomyolipomas; a benign tumour occurring chiefly in the kidneys. Although benign, the tumours can enlarge causing symptoms and those over $4 \mathrm{~cm}$ are prone to haemorrhage which can result in nephrectomy. LAM can occur in isolation (sporadic LAM) or associated with tuberous sclerosis complex (TSC-LAM). Treatment guidelines for angiomyolipoma tend to be based on retrospective series often from patients with TSC. The natural history of angiomyolipoma in sporadic LAM is not well understood.

Aims To document the natural history, risk factors for, and clinical features associated with, angiomyolipoma in a national cohort of patients with sporadic and TSC-LAM to plan clinical screening programs.

Methods Demographic data, clinical features, lung function and tumour size were obtained from clinical records of patients attending the National Centre for LAM in Nottingham. Tumour size was recorded as the longest tumour diameter on any imaging modality. Tumours smaller than $10 \mathrm{~mm}$ were assigned a value of $10 \mathrm{~mm}$. Ethical approval for use of patient data was obtained and patients gave informed consent.

Results 122 patients with definite or probable LAM by European Respiratory Society criteria were identified. Of these, 68 (56\%) had at least one angiomyolipoma. Angiomyolipoma were present in 50\% of patients with sporadic LAM and all patients with TSC-LAM Those with sporadic LAM tended to have smaller tumours than those with TSC-LAM (31 mm (SD 27) vs. 49mm (SD 27) respectively), were less often bilateral (40 vs. $79 \%$ ), were less likely to bleed (17 vs. $31 \%$ ) and less likely to require surgery or embolisation (49 vs. 66\%). Serial tumour measurements were available from some patients, tumours from those with TSC-LAM grew at 3.8 $\mathrm{mm} /$ year ( $\mathrm{n}=6$, SD 14.2) tumour growth in sporadic LAM growth was $4.3 \mathrm{~mm} /$ year ( $\mathrm{n}=16$, SD 8.2. $\mathrm{p}=0.46$ ).

Conclusions Angiomyolipomas are common in patients with sporadic and TSC-LAM. The tumours require intervention more frequently in TSC than sporadic LAM. The two patient groups may require different angiomyolipoma management. 